\title{
Les pratiques transnationales des immigrés chinois et marocains de Belgique
}

The Transnational Practices of Chinese and Moroccan Immigrants in Belgium Las prácticas transnacionales de los inmigrantes chinos y marroquíes en Bélgica

Marco Martiniello et Hassan Bousetta

\section{CpenEdition}

Édition électronique

URL : https://journals.openedition.org/remi/4512

DOI : $10.4000 /$ remi.4512

ISSN : $1777-5418$

Éditeur

Université de Poitiers

Édition imprimée

Date de publication : 1 novembre 2008

Pagination : 45-66

ISBN : 978-2-911627-49-0

ISSN : 0765-0752

Référence électronique

Marco Martiniello et Hassan Bousetta, «Les pratiques transnationales des immigrés chinois et

marocains de Belgique », Revue européenne des migrations internationales [En ligne], vol. 24 - n² | 2008, mis en ligne le 01 novembre 2011, consulté le 14 avril 2022. URL : http://journals.openedition.org/ remi/4512 ; DOI : https://doi.org/10.4000/remi.4512 


\title{
Les pratiques transnationales des immigrés chinois et marocains de Belgique
}

\author{
Marco MARTINIELLO* et Hassan BOUSETTA**
}

Depuis les années 1990, la littérature anglo-saxonne sur le transnationalisme s'est considérablement développée. Les avancées théoriques dans ce champ sont toutefois assez récentes même si les études de cas empiriques foisonnent déjà depuis de nombreuses années. En Europe continentale et plus particulièrement dans l'espace francophone, les débats théoriques sur les questions du transnationalisme n'ont également éveillé l'intérêt des chercheurs que tardivement. On peut faire le constat que les recherches empiriques consacrées aux pratiques transnationales des populations immigrées et issues de l'immigration continuent à être insuffisantes à ce jour.

L'un des objectifs de cet article est précisément de contribuer à combler cette lacune relative en nous concentrant sur le cas de la Belgique. De manière plus précise, et afin d'aller plus loin que les recherches européennes consacrées aux dimensions politiques et idéologiques du transnationalisme, notre objectif est ici de nous focaliser sur le transnationalisme de base («grassroot transnationalism »). En effet, dans l'optique d'approfondir la connaissance de ce concept en vogue, il nous est d'emblée apparu nécessaire de commencer par procéder à une description ethnographique la plus complète possible des pratiques transnationales des immigrés et de leurs descendants. Ce premier temps était indispensable afin de pouvoir identifier empiriquement les caractéristiques et les tendances les plus significatives de ces pratiques. En l'état actuel de la recherche sur ce sujet en Belgique, mais plus généralement aussi en Europe, l'élaboration d'hypothèses explicatives quant à l'émergence et la reproduction des

* Directeur de recherches au Fonds National de la Recherche Scientifique, directeur du Centre d'études de l'ethnicité et des migrations (CEDEM, Institut des Sciences Humaines et Sociales, Université de Liège), M.Martiniello@ulg.ac.be.

** Chercheur qualifié au FNRS, chargé de cours adjoint à l'Université de Liège, directeur adjoint du CEDEM. Boulevard du rectorat, 7, 4000 Liège, Belgique, Hassan.Bousetta@ulg.ac.be. 
pratiques transnationales des immigrés ne pouvait se concevoir que dans un second temps. Le recueil d'un matériau empirique qualitatif permettant de décrire de manière la plus systématique possible les différentes pratiques transnationales des différents groupes d'immigrés en Europe et en Belgique reste en effet un passage obligé pour toute démarche de ce type. La première étape de la recherche présentée ici nous a amené à sélectionner deux groupes d'immigrés relativement contrastés et à tenter d'identifier des régularités dans leurs pratiques économiques, sociales, culturelles et politiques transnationales pour ensuite dégager des constats comparatifs entre les pratiques transnationales des collectifs chinois et marocains de Belgique.

Bien que l'objectif premier de cet article ne soit pas proprement théorique, deux éléments de notre ligne de départ doivent être explicités. En premier lieu, il convient d'indiquer que notre approche porte sur les pratiques transnationales de deux groupes de population que nous qualifions a priori d'immigrés et non de communautés transnationales. En d'autres termes, notre approche rejette la distinction habituelle dans la littérature sur le transnationalisme entre les communautés transnationales et celles qui ne le seraient pas. En effet, nous pensons que les individus et les groupes issus de la plupart des populations immigrées sont susceptibles, dans certaines conditions, de s'engager dans des pratiques transnationales (Martiniello et Lafleur, 2008). Aucune population immigrée n'est par nature une communauté transnationale. Le prétendre revient, d'une part, à endosser une approche inappropriée et essentialiste du transnationalisme et, d'autre part, à réifier les «communautés transnationales ». De notre point de vue, l'engagement dans des pratiques transnationales peut être considéré comme un moment éventuel, comme une potentialité, dont il convient précisément d'expliquer l'occurrence et la reproduction dans le parcours de certains immigrés et de ce qu'ils définissent comme leur « communauté ». Il convient également d'indiquer à titre liminaire que la définition des activités transnationales que nous adoptons est inspirée des travaux d'Alejandro Portes (2001). Il s'agit d'activités réalisées au-delà des frontières étatiques par des immigrés dans les domaines économique, socio-culturel et politique que ce soit à la fois dans le pays dit de résidence et dans le pays dit d'origine ou encore dans le pays dit de résidence et dans un pays tiers.

Il découle de ce qui précède que notre posture théorique se veut constructiviste plutôt que fondée sur une déterminisme culturel. La position culturaliste ne nous paraît pas féconde en ce qu'elle tend à l'essentialisation des groupes concernés. Rien ne semble indiquer qu'il faille chercher d'abord et surtout dans la culture distinctive des populations immigrées l'explication de l'apparition, du développement et du maintien de pratiques transnationales. Certes, il ne serait pas non plus sensé d'écarter d'emblée et totalement l'importance éventuelle du facteur ethno-culturel. Les conditions macroéconomiques et politiques sont, de notre point de vue, premières dans l'explication de l'émergence des pratiques transnationales, de leur maintien et de leur reproduction. Ainsi par exemple les politiques des États dits d'origine à l'égard de leurs émigrés, parfois appelés citoyens résidant à l'étranger, et les politiques des États dits de résidence à l'égard des immigrés peuvent ouvrir ou fermer les opportunités d'apparition de pratiques transnationales. La culture et l'ethnicité n'apparaissent alors qu'en deuxième instance pour éventuellement éclairer de manière contingente la construction des formes que ces pratiques revêtent. 
D'un point de vue méthodologique, cette contribution s'attache aux pratiques transnationales de deux groupes très différenciés. Nous avons étudié, d'une part, une population immigrée déjà ancienne, les Marocains et leurs descendants qui sont de plus en plus souvent de nationalité belge et, d'autre part, la population immigrée chinoise dont l'implantation est dans l'ensemble plus récente. La recherche de terrain s'est étalée sur dix-huit mois, de janvier 2006 à juin 2007. Une revue de la littérature existante et un relevé des données statistiques relatives aux populations marocaine et chinoise ont été réalisés. Ensuite, un ensemble de soixante-trois entretiens ont été conduits $^{1}$ (33 répondants chinois et 30 marocains) avec des acteurs transnationaux actifs dans la sphère économique, politique ou socio-culturelle. Des entretiens ont également été menés avec des experts-informateurs qui connaissent particulièrement bien ces deux populations ${ }^{2}$.

Le présent article synthétise les résultats obtenus à l'issue de cette recherche. Il commence par un bref aperçu démographique des immigrés chinois et marocains en Belgique et de leurs descendants et par une brève présentation des points clés de leur histoire migratoire. Les trois parties suivantes présentent respectivement les pratiques économiques, socio-culturelles et politiques transnationales dans lesquelles s'engagent des Chinois et des Marocains de Belgique. Enfin, les conclusions s'efforcent de dégager des constats comparatifs ainsi que des hypothèses en vue de recherches ultérieures.

\section{LES IMMIGRÉS CHINOIS ET MAROCAINS EN BELGIQUE : BREF APERÇU DÉMOGRAPHIQUE}

Selon l'Institut National de Statistiques, il y avait 80602 Marocains et 7452 Chinois en Belgique en 2005. Ensemble, ces deux groupes formaient $10 \%$ de la population étrangère totale (Tableau 1). Ces chiffres doivent toutefois être considérés

Tableau 1 : Étrangers résidant légalement en Belgique par nationalités au $\mathbf{1}^{\mathrm{er}}$ janvier 2005

\begin{tabular}{|c|r|}
\hline Population totale & 10445852 \\
\hline Belges & 9574990 \\
Étrangers & 870862 \\
\hline dont ressortissant de pays tiers à l'UE & 291276 \\
\hline Maroc & 80602 \\
Turquie & 40403 \\
Congo (R.D.C.) & 13423 \\
USA & 11476 \\
Algérie & 7495 \\
Chine & 7452 \\
Roumanie & 5632 \\
\hline
\end{tabular}

Source : Registre National - Institut National de Statistiques, 2005.

1 Les entretiens ont été conduits respectivement par Merouane Touali pour les Marocains et Haina Lu pour les Chinois.

2 Le tableau récapitulatif du profil des personnes interviewées est disponible dans le rapport de recherche écrit par Haina Lu et Merouane Touali. Ce rapport est disponible sur demande. 
avec prudence. Il est en effet important de prendre en considération le nombre élevé des naturalisations enregistrées au cours des deux dernières décennies au sein de ces deux collectifs : 154846 Marocains et 2983 Chinois sont devenus belges entre 1991 et 2004. En ce sens, la décroissance de la population étrangère observable en Belgique au début des années 2000 est plus virtuelle que réelle (voir Figure 1).

Figure 1 : Immigration marocaine, turque, polonaise et chinoise en Belgique (1975-2004)

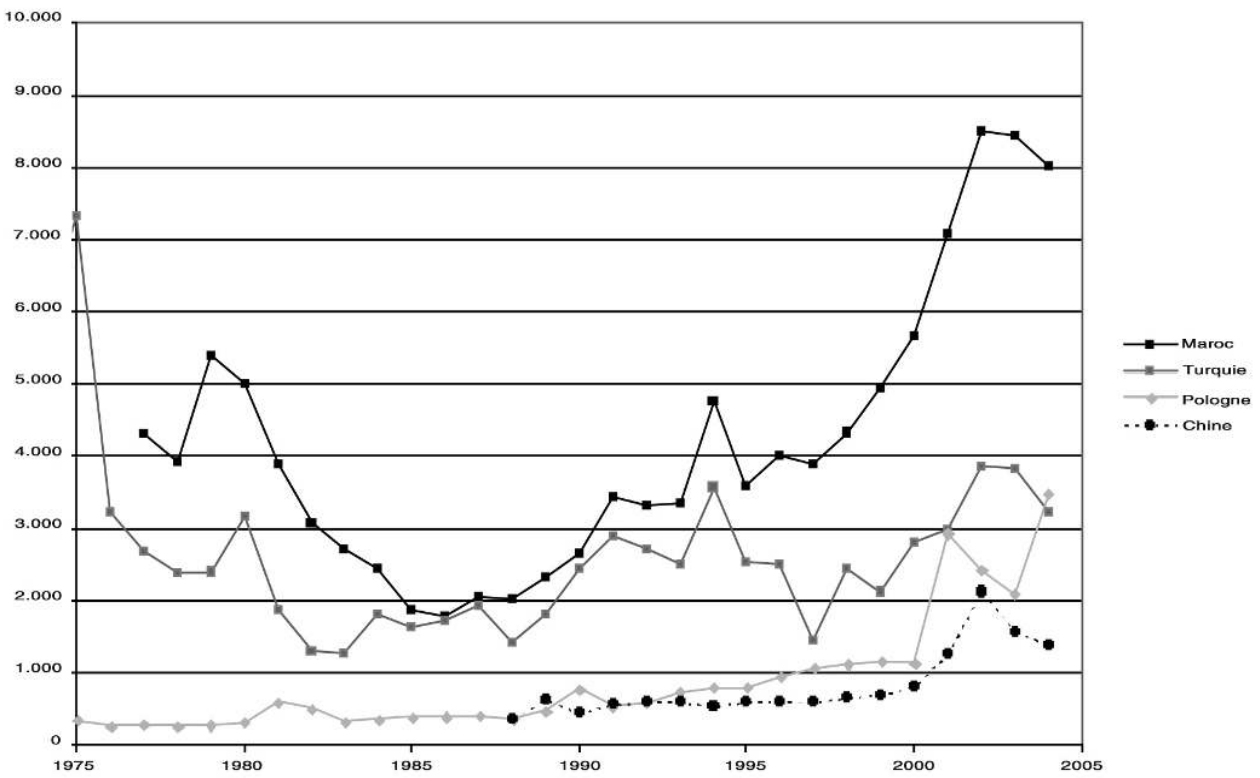

Source : Registre National - Institut National de Statistiques, 2005.

On trouve trace d'une présence chinoise en Belgique dès le XIX siècle. Ce sont surtout des marins de Guangdong et de la province de Fujian ainsi que des marchands de Qingtian et Wenzhou (Province de Zhejiang) arrivés via la France et l'Allemagne. C'est toutefois à partir des années 1950 que se développe une présence chinoise durable en Belgique ${ }^{3}$. Au regard de la politique restrictive en matière d'émigration mise en place par la République populaire de Chine après 1949, un grand nombre des Chinois qui arrivent en Belgique jusque dans les années 1980 proviennent en réalité de Hong Kong et de l'Asie du sud Est. Ceux qui arriveront par la suite de Chine continentale à partir des années 1980 seront essentiellement originaires des provinces de Zhejiang et Fujian. Ces vagues migratoires distinctes permettent d'établir une différenciation historique et sociologique entre une communauté de vieille implantation plutôt bien établie dans la restauration, et les nouveaux migrants chinois

3 La Belgique recense 371 personnes titulaires d'un passeport de Taiwan. 
présentant des profils socio-économiques plus bas. Enfin, il faut signaler qu'à ces nouveaux migrants s'ajoute une population difficile à estimer, mais en forte croissance, d'étudiants et de sans-papiers.

Comparativement, la présence marocaine en Belgique est nettement plus importante sur le plan quantitatif. Les premières traces de cette immigration sont recensées dans l'entre-deux-guerres. Cependant la présence en Belgique des Marocains en nombre significatif n'apparaît qu'après la signature de l'accord bilatéral de maind'oeuvre entre la Belgique et le Maroc en 1964. Cet accord marque le début d'un long flux d'immigration marocaine encore actif aujourd'hui et composé au départ essentiellement de travailleurs et de leurs familles. De nouveaux profils de migrants alimenteront également la croissance de la communauté marocaine, dont notamment les bénéficiaires du regroupement familial et un grand nombre d'étudiants qui s'établiront définitivement en Belgique au terme de leurs études. Comme l'indique la Figure 1, l'immigration en provenance du Maroc reste un phénomène actif. Chaque année un nombre important de Marocains obtiennent un permis de séjour, notamment par la voie du regroupement familial ou de la constitution de familles. La communauté marocaine de Belgique est donc simultanément dans un schéma migratoire, au regard du nombre important de nouveaux arrivants, et post-migratoire, au regard de l'ancienneté de l'implantation de la majorité de ses membres.

\section{LES PRATIQUES ÉCONOMIQUES TRANSNATIONALES}

Notre travail de terrain met en évidence des pratiques économiques transnationales de la part des Chinois de Belgique qui prennent notamment la forme d'investissements dans différents secteurs de l'économie tant de la Chine continentale que de Hong-Kong (industrie manufacturière, immobilier, restauration et même agriculture). La différenciation des dimensions économiques et sociales est parfois malaisée. C'est notamment le cas pour l'un de nos répondants, un entrepreneur sinobelge qui a investi dans la culture biologique du thé dans la perspective de venir en aide aux paysans pauvres d'une région de Chine continentale. Des Sino-Belges ${ }^{4}$ jouent aussi un rôle important dans le commerce entre la Chine et la Belgique voire d'autres pays européens. Les activités d'import-export sont importantes et concernent des produits aussi variés que les meubles, le textile d'habillement, les diamants, le chocolat, le vin, le matériel informatique, l'alcool, etc. Les entrepreneurs Sino-Belges se posent ainsi clairement en intermédiaires commerciaux entre les deux pays, voire plus largement entre la Chine et l'Union européenne.

Les trois exemples suivants illustrent la diversité des pratiques transnationales économiques de certains Chinois de Belgique. Le premier concerne une Chinoise active dans l'importation de vêtements de Chine, essentiellement à destination du

4 Cette expression ne renvoie ni à la (aux) nationalité(s) de ces personnes, ni à leur identité. Elle désigne l'ensemble des personnes actuellement établies en Belgique et qui sont d'origine chinoise ou chinoise. Nombre de ces personnes, mais pas toutes possèdent la nationalité Belge. 
marché belge et néerlandais, mais aussi d'autres pays européens comme l'Espagne, la Grèce et l'Allemagne. Elle travaille avec un partenaire chinois de Hong-Kong dont une partie de la famille est établie sur le continent et confectionne les vêtements. En 1992, elle décida d'investir dans l'immobilier en Chine. Ce projet lui fit contre toute attente perdre beaucoup d'argent.

Le second exemple concerne une Sino-Belge qui a commencé par posséder un restaurant chinois à Vienne à la fin des années 1980. En 1992, avec l'aide d'amis chinois basés en Italie, elle se lance dans l'importation de produits alimentaires pékinois à destination de l'Autriche. Elle commence ainsi à collaborer avec de grandes firmes chinoises à partir du milieu des années 1990. Cela va notamment l'amener à ouvrir une branche d'une chaîne de supermarchés pékinois en Autriche en même temps qu'elle commence à exporter des machines-outils européennes vers la Chine. Ce n'est qu'en 2002 qu'elle s'installe en Belgique pour ouvrir une filiale d'une grande chaîne de supermarchés de Shanghai.

Enfin, le troisième exemple est celui de ce propriétaire de sept restaurants chinois en Belgique qui a décidé d'investir à Shanghai en 1992. Durant trois ans, il a été à la tête d'une usine de transformation de coton. Cette aventure s'est terminée par une faillite. Dès 1995, il s'est à nouveau tourné vers la restauration en ouvrant cinq établissements, dont un à Shanghai et un à Pékin. Puis il investit dès 2000 dans la construction d'appartements pour la vente à des particuliers dans différentes villes de Chine. Dans la réalisation de toutes ces opérations, il a été secondé par le même partenaire chinois établi au pays.

Ces exemples que nous pourrions multiplier montrent un dynamisme économique transnational incontestable des Chinois établis en Belgique. Il est toutefois difficile sur la base de la présente recherche de proposer une estimation du volume et de la valeur monétaire de ces échanges. Quoique nous les ayons interrogés sur ce sujet, nos répondants se sont montrés peu prolixes en la matière. En revanche, nous avons pu dégager une série de traits communs à ces pratiques et à ces acteurs économiques transnationaux sino-belges.

En premier lieu, il faut faire le constat qu'un grand nombre d'entrepreneurs transnationaux sino-belges ont été à un moment de leur parcours, ou le sont encore, actifs dans le secteur de la restauration en qualité de propriétaire et/ou de gérant d'un ou de plusieurs restaurants chinois. Et si de nouveaux migrants chinois sont nombreux à souhaiter monter leur propre restaurant, on peut également constater que de nombreux propriétaires de ce type de commerce souhaitent également en sortir pour diversifier leurs activités entrepreneuriales. Si l'on ne peut nier que des éléments de type culturel comme les valeurs et la structure familiale jouent un rôle dans l'explication du développement et du succès de ces restaurants, ils n'expliquent en rien le choix de ce type d'activité commerciale au départ. Des variables, renvoyant aux conditions sociales, économiques et politiques, semblent avoir un impact plus déterminant que la culture sur cette décision (Leung, 2004). Lorsque ces conditions objectives changent, les choix entrepreneuriaux tendent à changer aussi. En réalité, la 
transnationalisation des activités économiques de cette population est très souvent le fruit de changements dans leur environnement dans les sociétés d'immigration.

En second lieu, nous constatons que la plupart des personnes interviewées se sont lancées dans des activités économiques transnationales au début des années 1990 et que ce mouvement s'est intensifié au début des années 2000. L'accélération de la mondialisation des échanges économiques et l'ouverture de la Chine à l'économie de marché et aux investissements étrangers ne sont pas étrangers à ce mouvement. Le développement des pratiques économiques transnationales des immigrés chinois a accompagné l'ouverture de nouvelles opportunités économiques dans le pays d'origine. D'un point de vue strictement économique, il devenait plus intéressant — en réalité il devenait tout simplement possible — pour les Chinois de l'étranger d'investir en Chine. Le marché de l'immobilier qui a explosé en Chine depuis le début des années 1980 a par exemple attiré de nombreux investisseurs Chinois établis en Belgique et ainsi contribué à la transnationalisation de leurs activités.

En troisième lieu, si les activités économiques transnationales des Sino-Belges concernent principalement la Chine et la Belgique, elles tendent aussi à couvrir d'autres pays européens et parfois même les USA. D'une manière générale, il semble bien que la Chine continentale se taille la part du lion en termes d'investissements, au détriment notamment de Hong-Kong.

En quatrième lieu enfin, les Chinois de Belgique engagés dans des pratiques économiques transnationales semblent tous appartenir à la première génération. Les générations suivantes (deuxième et troisième) ne semblent pas pour l'instant actives sur le terrain économique en Chine même si certaines de nos données d'entretiens révèlent chez les nouvelles générations un intérêt «a priori » pour un investissement dans ce qu'ils qualifient comme le «pays des ancêtres ».

Au-delà de ces quatre traits communs aux pratiques et aux acteurs économiques transnationaux sino-belges, les entretiens mettent en lumière trois facteurs explicatifs de l'engagement dans ce type de pratiques : les motivations individuelles, les compétences, les ressources des acteurs et les conditions objectives.

L'ambition personnelle, la volonté de saisir de nouvelles opportunités et de réaliser des profits, sont les motivations individuelles principales à la base de l'engagement dans des pratiques économiques transnationales. Une certaine littérature sur le transnationalisme chinois (Nyiri, 2005 ; Leung, 2004) inspirée des travaux d'Appadurai (1996) considère que cet engagement aurait pour objectif la construction d'une identité supranationale permettant de relier les Chinois à leur diaspora à travers le monde. Cette quête identitaire particulièrement adaptée au monde contemporain serait un objectif en soi poursuivi au travers des activités transnationales. Rien ne permet à partir de nos entretiens de soutenir cette hypothèse. Le développement d'un sentiment d'appartenance multiple semble davantage être la conséquence des activités transnationales que sa motivation première. 
En ce qui concerne les compétences et les ressources des acteurs, le niveau d'éducation ne semble pas jouer un rôle important pour expliquer l'engagement dans les pratiques économiques transnationales. Les entrepreneurs sino-belges ont des niveaux d'éducation variables, allant du certificat d'études primaires à la thèse de doctorat. Il est, de plus, assez surprenant de constater qu'il n'est pas indispensable d'être expérimenté dans les nouvelles technologies de la communication pour se lancer dans la conduite d'activités économiques transnationales. Les entrepreneurs de la première génération, qui sont majoritaires, continuent à utiliser prioritairement la téléphonie classique plutôt que les nouveaux outils de communication informatiques.

La possession et la disponibilité d'un capital de base à investir est, comme dans toute activité entrepreneuriale, une condition fondamentale pour élargir géographiquement son rayon d'action. En l'occurrence, les Chinois commencent souvent par mobiliser un capital personnel ou familial avant de chercher par la suite à obtenir des emprunts auprès des banques belges.

Au-delà de leur capital économique, les entrepreneurs chinois de Belgique doivent également mobiliser des ressources sociales pour espérer poursuivre avec succès des activités économiques transnationales. Ainsi, pouvoir compter sur des partenaires fiables en Chine est fondamental non seulement pour nouer des contacts d'affaires mais également pour réduire le nombre de voyages entre la Chine et la Belgique. La littérature a mis en évidence l'importance de la mobilisation de réseaux ethniques pour les hommes d'affaires chinois de la diaspora (Forschauer, 1997 ; Mitchell, 1995 ; Ong et Nonini, 1997). Nos données confirment ce constat. Les entrepreneurs sino-belges jouent sur les réseaux ethniques dans leurs activités transnationales. Nous ne sommes toutefois pas en mesure d'indiquer s'il y a une particularité chinoise à ce propos. Par ailleurs, les réseaux d'affaires des Sino-belges dépassent aussi souvent le réseau ethnique. Nyri (2005) indique enfin que les liens personnels (guan xi) caractérisent le business chinois. De nombreuses personnes interrogées confirment que pour mener des affaires à bien en Chine, il faut souvent de bonnes relations, notamment avec des représentants de l'État et du Parti Communiste.

Si la connaissance des langues nationales belges ne semble jouer aucun rôle, en revanche le statut juridique du séjour des immigrés chinois est crucial. Ainsi, posséder un passeport Belge ou européen permet à l'homme d'affaire de bénéficier de politiques avantageuses en matière fiscale et de traitements préférentiels. Il n'est donc guère surprenant de constater que les entrepreneurs chinois sont nombreux à avoir acquis la nationalité belge, ou une autre nationalité européenne au gré de leur parcours migratoire antérieur.

Enfin, il faut souligner que des conditions objectives en Chine et en Belgique peuvent réellement modeler les activités économiques transnationales. En Belgique, le secteur de la restauration chinoise a par exemple connu des difficultés liées aux changements dans la politique belge d'immigration. Ainsi, la lutte contre l'immigration clandestine rend plus difficile l'utilisation d'une main-d'oeuvre en provenance de Chine très peu payée. De plus, une politique d'immigration restrictive a rendu de plus en plus difficile le recrutement de chefs coqs en Chine. Enfin, la régularisation menée 
par le gouvernement belge en 1999 et 2000 a permis à un certain nombre d'immigrés chinois d'obtenir un permis de séjour définitif. Cela a conduit à la création de nouveaux restaurants chinois et à l'accroissement de la concurrence et de la pression dans ce secteur déjà proche de la saturation. Dans ces conditions, certains entrepreneurs ont cherché soit à en sortir, soit à se diversifier, notamment en recherchant de nouvelles opportunités dans le champ économique transnationale.

Les changements dans les conditions économiques et politiques objectives de la Chine contemporaine ont aussi encouragé le développement de pratiques économiques transnationales. Particulièrement après les événements de la place Tienanmen, le gouvernement s'est montré très persuasif avec les diasporas chinoises afin de les convaincre de rapatrier les investissements nécessaires au développement du pays. Les autorités locales ont parfois invité les Chinois de la diaspora à aider financièrement la construction d'infrastructures collectives comme des écoles dans les régions pauvres du pays. De manière plus subtile, le gouvernement central et certaines autorités locales organisent des événements spécifiques en direction de la diaspora comme la conférence mondiale des entrepreneurs chinois. Il s'agit d'espaces de rencontres en vue de nouer des affaires et qui servent de support objectif à la croissance des activités économiques transnationales des Chinois. D'une certaine manière, le gouvernement chinois peut tantôt limiter, voire empêcher les pratiques économiques transnationales, tantôt les susciter lorsque son intérêt le commande.

Les pratiques économiques transnationales des migrants marocains se structurent de manière différente et résultent des modes de vie et des structures de la communauté marocaine en Belgique. Pendant longtemps, les circuits d'échanges économiques entre le Maroc et la Belgique ont fonctionné à sens unique, du pays d'immigration vers le pays d'origine (transferts d'épargne, investissements immobiliers, projets agricoles, etc). La situation actuelle est marquée par des échanges économiques beaucoup plus soutenus et fonctionnant dans les deux directions.

Les transferts d'épargne constituent le thème le plus significatif du transnationalisme économique des Marocains, compte tenu du volume des sommes envoyées au Maroc depuis la Belgique. C'est la matérialisation la plus évidente des liens qu'entretiennent les migrants marocains avec leur pays d'origine. Les transferts d'épargne des migrants marocains sont initialement le fait d'individus et de ménages. Ce sont de petites sommes au départ qui finissent par devenir des flux financiers importants à l'arrivée. Ils ont souvent une importance vitale pour les familles restées au pays et revêtent également une importance stratégique pour l'État marocain alors qu'ils représentent la première recette de la balance des paiements du pays, bien avant le tourisme et les investissements étrangers directs ${ }^{5}$. En 1999, la Belgique ${ }^{6}$ arrivait en

5 Ils ont atteint un record historique en 2006 avec 40,7 milliards de MAD soit un peu moins de 4 milliards d'Euros. Et les chiffres de l'Office Marocain des Changes publiés fin février 2007 font état de nouvelles perspectives de croissance de ces remises (www.oc.gov.ma). Cela représente près de $10 \%$ du PIB du pays et plus du quart des dépôts des banques commerciales.

6 C'est l'Union Economique Belgo-Luxembourgeoise qui est en réalité prise en considération ici. 
troisième position parmi les pays émetteurs de transferts de fonds vers le Maroc avec 1,08 milliard de MAD. En 2003, elle reculait à la quatrième place derrière l'Espagne. Proportionnellement, le volume des transferts d'épargne depuis la Belgique est pourtant en légère croissance sur la période 1999-2003, passant de 5 à $6 \%$ du total de l'ensemble des transferts effectués vers le Maroc.

La pratique du transfert d'épargne des immigrés marocains tout au long de l'année a conduit à l'organisation de tout un secteur spécialisé dans le transfert de fonds. Les banques marocaines ont investi massivement dans ce type d'opérations depuis de très nombreuses années, mais des officines privées se sont également positionnées sur ce marché. Comme certains répondants nous l'ont confié, la multiplication des canaux de transfert et des produits bancaires marocains sur le territoire belge n'exclut toutefois pas le recours à des transferts très importants en cash pour des investissements au pays.

Historiquement, un grand nombre d'activités économiques transnationales se sont développées en Belgique pour répondre à l'émergence d'une demande de produits de consommation en provenance du pays d'origine. Les pratiques économiques transnationales qui se sont organisées autour de cette demande sont donc étroitement connectées aux modes de vie et de consommation des immigrés marocains en Belgique. La circulation de produits dits exotiques à travers le développement du commerce et des restaurants ethniques a contribué à la création et au renforcement d'un circuit économique entre la Belgique et le Maroc. Mais on peut également faire le constat que ces circuits économiques transnationaux marocains en Belgique se sont quelque peu autonomisés de la communauté marocaine stricto sensu pour cibler l'ensemble de la population d'origine arabe et/ou musulmane et parfois même la clientèle belge.

Si les activités transnationales consistant à distribuer et commercialiser des produits de consommation originaires du pays d'origine se sont fortement développées, on assiste également au renforcement des activités visant à investir dans des projets d'activités économiques dans le pays d'origine. C'est un phénomène qui touche également la jeune génération et qui implique la mobilisation de compétences professionnelles acquises dans la société d'immigration. Ce sont en règle générale des projets de petite taille ou de taille moyenne. Certains de ces projets ont un lien direct avec l'activité dans le pays de résidence. C'est le cas de cet investisseur migrant marocain spécialisé dans la vente de produits alimentaires en Belgique qui a investi dans une unité de conditionnement de fruits secs dans sa région d'origine en vue d'exporter vers le marché belge.

Les motivations des projets d'investissement dans le pays d'origine sont multiples. Certains répondants avancent l'avantage économique que leur procure leurs liens familiaux, d'autres la volonté de préparer leur retraire dans le pays d'origine, d'autres encore espèrent profiter des opportunités d'investissement liées à l'ouverture économique actuelle du Maroc. Dans la plupart des cas, les répondants avancent qu'ils éprouvent des craintes quant à la mise en œuvre du projet notamment au niveau des obstacles administratifs. 
La mobilité est une question importante dans le développement d'activités économiques transnationales. S'il existe bien des formes de transnationalisme sans mobilité physique des acteurs, la plupart des projets économiques impliquent une présence régulière à la fois dans le pays d'origine et de résidence. Les entrepreneurs marocains évoquent la nécessité d'une présence sur place pour la conduite de leurs projets d'activités économiques et la nécessité d'effectuer des déplacements fréquents comme une contrainte supplémentaire.

Dans cette logique, il est intéressant de se pencher sur la localisation géographique des investissement des immigrés porteurs de pratiques économiques transnationales avec le Maroc. Bien qu'il soit difficile de tirer des enseignements de portée générale à ce sujet, nos répondants n'affirment pas avoir investi prioritairement dans leur région d'origine, à l'exception de l'investissement immobilier. Dans la période actuelle, marquée par une grande ouverture de l'économie marocaine à l'investissement étranger, il n'est pas étonnant de constater que la rentabilité économique est un critère très présent dans le discours des acteurs, lequel prend l'ascendant sur l'attachement à la région d'origine dans la décision d'investir.

Les données du terrain montrent toutefois que la localisation des activités économiques transnationales dans les régions d'origine fonctionne également comme un mécanisme visant à sécuriser la mise en œuvre des projets. En effet, le modus operandi de certains projets économiques transnationaux des Marocains de Belgique, que nous avons pu observer, montre l'importance de l'intersection entre logiques économiques et logiques familiales. Incontestablement, les réseaux familiaux, voire sociaux, offrent un point d'appui structurant aux pratiques économiques transnationales. Certains répondants développent de petits projets uniquement grâce à la collaboration active des membres de la famille restée au pays. D'autres utilisent leurs réseaux familiaux, amicaux et sociaux pour rechercher les meilleures opportunités d'investissement.

\section{LES PRATIQUES SOCIALES ET CULTURELLES TRANSNATIONALES}

Les pratiques sociales et culturelles transnationales des Chinois de Belgique sont extrêmement diversifiées. Elles peuvent être regroupées en quatre catégories : les pratiques académiques, artistiques, d'aide dans la lutte contre la pauvreté ou dans le cas de catastrophe naturelle et les pratiques religieuses.

Bien que les scientifiques chinois établis en Belgique ne représentent qu'une infime proportion de la communauté, ils entretiennent des liens étroits avec les institutions académiques et leurs homologues en Chine. Ils s'y rendent régulièrement pour donner des conférences, assister à des séminaires ou des colloques. Ils publient dans des revues scientifiques chinoises. Certains y vont en qualité de professeur invité comme, par exemple, ce chercheur dans un hôpital universitaire belge qui est retourné dans l'université dans laquelle il avait étudié avant son départ. Son objectif avoué était d'aider cette institution et ses collègues en transférant son savoir acquis en Belgique. 
Les pratiques artistiques observées concernent la littérature et la peinture. Ainsi, ce répondant, romancier et poète, a-t-il écrit un livre sur l'expérience chinoise en Belgique. Il est surtout l'auteur d'une série de trois romans sur la Chine de la Révolution culturelle. Il a reçu plusieurs prix et récompenses pour son oeuvre en Chine où une partie de ses écrits est publiée. Il est en relation étroite avec des écrivains chinois et il se rend souvent au pays pour assister à des séminaires. Bien que connu et réputé dans les cercles littéraires chinois, les rentrées financières de son activité d'écrivain ne suffisent pas. C'est la raison pour laquelle il est propriétaire d'un restaurant chinois en Belgique.

Quelques peintres Chinois de Belgique ont organisé des expositions en Chine mais aussi dans d'autres pays asiatiques et européens. Ils donnent aussi des conférences en Belgique, en Chine et aux Pays-Bas. Un couple de peintres affirme clairement sa volonté de favoriser les échanges culturels entre la Chine et la Belgique. Il a donc créé une ONG et organisé de nombreux événements artistiques dans les deux pays : une exposition en Belgique en 1996 qui présentait les oeuvres d'artistes chinois établis dans 16 pays différents, une exposition de bande dessinée chinoise en 2004 à Bruxelles, etc. Il a aussi publié plusieurs livres en Chine. L'époux est par ailleurs professeur invité dans une université chinoise. Le couple a géré pendant plusieurs années un restaurant chinois en parallèle à son activité artistique qui peu à peu est devenue une source de profit économique. Clairement une pratique culturelle peut aussi avoir une importante dimension économique.

Les pratiques d'aide au développement, d'aide dans la lutte contre la pauvreté ou dans le cas de catastrophe naturelle sont nombreuses dans la communauté chinoise de Belgique. Des dons sont récoltés par des associations chinoises qui souvent les transfèrent à l'ambassade de Chine qui fait elle-même suivre à des organisations en Chine selon un système dont la transparence ne semble pas être la caractéristique principale. Ces dons peuvent servir des causes diverses comme la construction d'écoles dans des régions pauvres du pays ou l'aide directe aux paysans.

Deux organisations religieuses chinoises sont aussi fortement impliquées dans des pratiques transnationales. Il s'agit de l'église protestante évangélique de Hong Kong et de la lumière de Bouddha (Fokuangshan) originaire de Taiwan. Ces deux organisations ont des liens avec des associations chinoises avec lesquelles elles mettent sur pied des événements culturels. Fokuangshan Belgique est une des 100 branches de Fokuangshan International disséminées dans le monde. Elle entretient des contacts étroits avec les instituts bouddhistes de Chine continentale et recolte des dons pour la construction d'écoles en Chine. Elle possède sa propre maison d'édition et diffuse une lettre d'information dans différentes langues. En Belgique, elle regrouperait environ 200 personnes.

Une autre dimension de la vie sociale et culturelle transnationale mérite d'être soulignée. Il s'agit des organisations chinoises pan-européennes qui sont bien implantées en Belgique. L'union des associations chinoises en Europe est la plus importante. Elle organise depuis une quinzaine d'années des sessions dans différentes 
villes d'Europe en vue de promouvoir la coopération commerciale et scientifique entre la Chine et l'Europe et aussi l'intégration des Chinois dans les sociétés européennes tout en renforçant la solidarité entre les Chinois établis dans les différents pays européens. Ces sessions annuelles regroupent jusqu'à 600 Chinois d'Europe. Des personnalités politiques chinoises n'hésitent pas à faire le déplacement de Chine pour y assister, ce qui prouve l'importance de ce type d'organisation pour la Chine.

Tout comme les activités économiques transnationales, les activités sociales et culturelles transnationales ont commencé vers le début des années 1990 pour s'intensifier dans les années 2000. Comme nous venons de le voir, elles couvrent la Chine et la Belgique, mais elles présentent une dimension pan-européenne importante. Enfin, elles sont principalement conduites par des Chinois de la première génération même si, depuis quelques années, des Chinois de la seconde génération s'efforcent de renouer avec leurs racines en voyageant dans la région d'origine de la famille.

Contrairement aux activités économiques transnationales, les acteurs engagés dans des pratiques sociales et culturelles transnationales mettent en avant des motivations plus diverses et plus désintéressées. Ceux qui s'engagent dans des projets sociaux disent le faire par obligation morale envers la Chine. Les acteurs culturels mettent plutôt en avant les valeurs de l'échange culturel, même si certains reconnaissent que leurs activités peuvent avoir des conséquences économiques et professionnelles en leur faveur.

En ce qui concerne les compétences et les ressources des acteurs sociaux et culturels transnationaux, elles ne se distinguent fondamentalement guère de ce que vous avons observé à propos des pratiques économiques. On remarque notamment la même corrélation entre l'engagement dans les pratiques transnationales et un bon niveau économique.

De même, les conditions objectives liées aux pays d'origine et de résidence jouent un rôle important dans la promotion des pratiques sociales et culturelles transnationales. Tant la Chine que Taiwan ont une politique proactive envers les Chinois d'Outre-mer. Le gouvernement chinois s'efforce de perpétrer chez eux l' « amour de la Chine » via notamment la promotion de l'enseignement de la langue et de la culture chinoises. L'ambassade chinoise en Belgique aide aussi les associations à inviter des artistes chinois lors de fêtes traditionnelles. Quant au gouvernement belge, il n'a pas de politique spécifique envers la communauté chinoise même si les autorités locales belges l'aident parfois dans la mise en œuvre de manifestations ponctuelles. La ville d'Anvers a par exemple mis un terrain de camping gratuit à la disposition de Fokuangshan pour une de ses activités.

En ce qui concerne les Marocains, leurs pratiques sociales et culturelles transnationales sont également d'un grand dynamisme et ont acquis une certaine visibilité. Un nombre considérable d'initiatives a vu le jour à travers le développement du travail des associations volontaires. Celles-ci conduisent non seulement des projets localisés, mais entretiennent également de larges réseaux de solidarité avec le pays 
d'origine. Un ensemble d'acteurs associatifs issu de l'immigration marocaine en Belgique s'est constitué qui entretient une dynamique de développement et de solidarité à travers une myriade de projets.

La première observation qu'il convient de faire est que les réseaux associatifs des immigrés marocains se sont fortement autonomisés par rapport à l'État marocain. Alors que durant les premiers temps de l'immigration, les autorités de Rabat cherchaient à maintenir un contrôle très strict sur les initiatives politiques, sociales et culturelles de la communauté, ce n'est plus le cas de manière aussi évidente aujourd'hui. Les projets de solidarité internationale des immigrés marocains se développent aujourd'hui sur le terrain dans des conditions de relative autonomie. Cela n'exclut toutefois pas le maintien de contacts réguliers avec les pouvoirs publics. Les projets évoqués par nos répondants évoquent de muliples exemples de collaboration avec les autorités locales tant au niveau de la logistique que de la mise en oeuvre des actions. Ils mentionnent également le climat renouvelé dans lequel se déroule cette coopération. Certains répondants observent même que les contacts avec les autorités préfectorales marocaines, si difficiles dans les années antérieures et bien souvent troublés par des pratiques de corruption, se sont améliorés.

Une forme d'appartenance à distance au pays d'origine est mobilisée dans leur discours sans que celle-ci ne prenne nécessairement les formes d'un discours patriotique. Ces démarches se veulent avant tout pragmatiques. Certains répondants disent même que leur pays d'origine n'est pas le seul où ils sont actifs dans le domaine de la solidarité internationale.

Si le patriotisme au sens fort est absent, les projets relatifs aux grands chantiers de développement poursuivis par le Souverain et son gouvernement sont par contre évoqués. Un nombre significatif de nos répondants font ainsi référence à l'Initiative de Développement Humain promue par les autorités du pays. Ce programme lancé en mai 2005 par le Roi Mohamed VI vise à combattre la pauvreté et à promouvoir la création d'emploi. Des projets de nature très diverses, portés tant par des structures gouvernementales que par le secteur associatif, prennent place dans le cadre de cette initiative qui s'apparente à un chantier de mobilisation nationale. Les émigrés n'hésitent pas à redéfinir leurs projets par rapport au cadre de l'INDH. Fondamentalement, il s'agit le plus souvent de positionnements tactiques visant à rehausser l'utilité sociale d'initiatives privées pour mieux négocier le face à face avec l'administration locale et ses lourdeurs bureaucratiques.

La seconde génération s'investit également dans ces projets de développement. Leurs motivations s'inscrivent dans une perspective de mobilisation concernant l'amélioration des conditions de vie des catégories de la population marocaine avec lesquelles ils sont en contact (initiatives locales dans les villages d'origine, projets humanitaires, etc.). Leurs projets semblent se développer sur un terrain plus idéaliste. La protection de l'enfance via le soutien à des orphelinats ou l'éducation figurent parmi les exemples cités. L'un de nos répondants travaille au développement d'une structure d'éducation qui vise à acheminer des manuels scolaires, organiser des cycles de formation et animer des sessions pédagogiques durant les 
périodes de vacances. Cette activité est étroitement liée à celle, menée auprès d'enfants de l'immigration marocaine en Belgique. L'activité transnationale se présente donc ici comme le prolongement géographique d'une mobilisation au sein de la société de résidence.

Le niveau de formation des acteurs influence fortement leurs actions. Les réseaux d'étudiants et de professionnels sont particulièrement éclairants de ce point de vue. L'association des biologistes marocains en Belgique, par exemple, tente de soutenir les jeunes chercheurs en biologie au Maroc dans leurs universités ou de faciliter leur mobilité. Par ses réseaux de contacts dans les universités européennes et nord américaines, elle cherche aussi à mieux équiper les laboratoires marocains. Ces réseaux spécialisés se sont développés depuis le milieu des années 1990 en parallèle avec la migration d'étude des Marocains. Par leur ancrage dans des circuits universitaires internationaux, ils ont permis un déploiement original de pratiques transnationales spécialisées et à haute valeur ajoutée.

Les réseaux transnationaux marocains sont aussi marqués par la dimension religieuse. Deux répondants particulièrement actifs évoquent leurs projets de soutien à la construction ou à l'aménagement de mosquées au pays. La dimension caritative à travers des mobilisations récurrentes pour l'acheminement de matériel médical est également présente chez cette catégorie d'acteur. Dans la pratique, les réseaux religieux se manifestent par leur capacité à mobiliser des fonds sans intervention institutionnelle ou associative. Ce sont les collectes organisées à l'issue des services religieux qui alimentent tant les projets en direction du Maroc que les activités de reproduction matérielle du culte en Belgique.

Bien que numériquement plus modestes, les Juifs marocains de Belgique se distinguent par le maintien de relations transnationales avec le Maroc. La valorisation du patrimoine culturel et artistique judéo-marocain auquel on assiste dans la capitale belge est rendue possible par le retissage de relations personnelles, associatives et institutionnelles de cette communauté avec le Maroc. L'ambition de préserver les lieux de mémoire de la communauté juive au Maroc (cimetières, mausolées, synagogues, etc.) sont à la base de nouveaux projets transnationaux chez ces acteurs qui, rappelonsle, restent très peu nombreux en Belgique.

\section{LES PRATIQUES POLITIQUES TRANSNATIONALES}

Les pratiques politiques transnationales explicites des Chinois de Belgique sont soit très rares soit difficilement identifiables. En tout état de cause, nous n'avons découvert qu'une petite poignée de Chinois engagés dans la politique chinoise. Une des personnes interviewées a dit être un membre actif du Parti Communiste chinois et participer à diverses réunions politiques au pays. Il existe bien une mobilisation en faveur de l'unification de la grande Chine, mais d'une manière générale le système politique chinois ne laisse que peu d'ouverture à une participation effective des Chinois de l'étranger. 
La situation est différente dans le cas de Taiwan. Les ressortissants taiwanais ont le droit de vote au pays, mais ils doivent y retourner pour l'exercer, ce qui coûte du temps et beaucoup d'argent. Les principaux partis politiques taiwanais sont présents en Europe, mais leur fonctionnement et leur importance restent d'un accès difficile.

Enfin, les organisations chinoises pan-européennes tentent parfois de se poser en interlocuteurs des institutions européennes afin de chercher à négocier des solutions aux divers problèmes rencontrés par les Chinois dans l'Union européenne. Notre recherche n'a toutefois pas permis d'approfondir cette dimension du transnationalisme politique chinois rarement médiatisée. En effet, mis à part lors du périple de la flamme olympique en Europe au cours duquel Chinois et Tibétains se sont affrontés, les Chinois font rarement la Une des médias comme acteurs politiques locaux ou transnationaux.

L'activité politique transnationale des Marocains de Belgique se décline par contre de différentes manières. Les pratiques identifiées permettent de dégager deux figures principales : les personnes actives en politique belge ou dans le monde associatif, qui maintiennent des liens avec la politique marocaine et les militants des partis politiques marocains en Belgique ${ }^{7}$. Le rapport de ces deux catégories d'acteurs à l'espace politique marocain est fortement conditionné par les ouvertures que le pays a progressivement accordées à la population émigrée. Même si les Marocains ne disposent pas du droit de vote à l'étranger, les institutions chargées d'encadrer ses demandes se sont multipliées depuis le début des années 1990. Les mécanismes de consultation se sont également institutionnalisés avec la création récente d'un Conseil supérieur de la communauté marocaine résidant à l'étranger. Les conditions de la participation à l'élaboration de la décision politique au Maroc, les dossiers des transferts d'épargne, de l'investissement économique, des conflits juridiques familiaux, de la sécurité sociale, de la migration, de l'encadrement religieux sont autant de questions qui font l'objet d'intenses activités transnationales.

Les mouvements de la gauche et de l'extrême gauche marocaine sont particulièrement dynamiques dans l'entretien de dynamiques de participation politique transnationale. Ces courants s'organisent sous forme de sections à l'étranger des partis politiques au Maroc. Ils sont structurés de manière relativement peu formelle, mais se sont développés de manière très active sur le terreau du mouvement associatif de l'immigration ou dans le syndicalisme. Présents depuis de longues années au sein de la société civile belge sur des dossiers comme ceux des sans-papiers, des droits de l'homme, de la Palestine, etc., ils perpétuent à l'étranger une longue tradition de militantisme, de résistance critique, voire d'opposition frontale, inaugurée sous l'ère du roi Hassan II. La composition sociologique de ces mouvements est fortement tributaire des migrations d'étudiants. Leurs animateurs orientés vers la scène politique marocaine sont le plus souvent constitués d'étudiants ou anciens étudiants ayant connu une socialisation politique sur les campus des universités marocaines. On retrouve ici peu de représentants de la seconde génération issue de l'immigration des travailleurs marocains.

7 On peut y ajouter l' implication directe dans la politique électorale marocaine. Ce cas est représenté par un répondant de la deuxième génération né en Belgique, y aillant grandi et y maintenant une résidence, mais élu adjoint au maire dans une municipalité marocaine. 
La présence politique des mouvements de gauche marocains est particulièrement sensible à Bruxelles à travers nombres d'activités publiques (débats, concerts, expositions, manifestations, etc.). Les militants entretiennent des contacts permanents avec leurs homologues dans les partis et mouvements de la gauche marocaine. Grands consommateurs des médias marocains, ces acteurs disposent d'une connaissance très fine des grands enjeux politiques auxquels le Maroc est confronté et les traduisent dans la réalité belge et dans la population immigrée. Les courants de la gauche et de l'extrême gauche marocaine en Belgique sont relativement morcelés et les clivages qui les structurent sont avant tout le reflet des lignes de divisions partisanes à l'œuvre au sein du champ politique marocain.

Les personnes actives dans la politique belge sont également présentes sur cette scène transnationale. Il faut à cet égard rappeler que depuis une dizaine d'années, la Belgique a connu de nombreuses percées électorales de candidats issus de l'immigration et que les Marocains ont été particulièrement actifs lors des campagnes électorales. On retrouve ainsi de nombreux élus belges d'origine marocaine dans les assemblées au niveau local, régional et national. Dans bien des cas, ces élus sont amenés à s'intéresser et même à prendre position face à des enjeux politiques transnationaux. Cet investissement n'a pas manqué de soulever dans le débat public des questions relatives aux conflits d'allégeance que ces prises de position impliquent. La participation de parlementaires belges aux travaux préparatoires à la création du Conseil supérieur de la communauté marocaine ont ainsi pu être présentés comme problématiques. Mais si le débat médiatique est resté limité, c'est d'abord parce que ces phénomènes ne touchent qu'une très faible minorité d'élus. En règle générale, il s'agit d'élus qui ont connu un parcours de militants associatifs et qui gardent un intérêt pour les processus de démocratisation du Maroc. Dans le contexte politico-électoral belge où la survie politique des candidats d'origine étrangère est fortement tributaire de leurs soutiens communautaires, on peut penser que la pratique transnationale ne vise pas uniquement à transformer le Maroc mais également à présenter à l'électorat local un profil d'attachement, voire de défenseur, des intérêts du pays d'origine. Cette posture est particulièrement évidente en ce qui concerne les prises de position concernant le Sahara Occidental. L'effet de levier qu'elles peuvent jouer sur le terrain local n'est jamais aussi visible que sur ce dossier. Dans ces conditions, on ne s'étonnera guère de constater qu'on ne connaît que très peu de Belgo-marocains actifs en politique qui se positionnent explicitement sur le sujet en rupture avec la position officielle de Rabat visant à affirmer la marocanité du Sahara.

\section{POUR CONCLURE : QUELQUES CONSTATS COMPARATIFS ET PERSPECTIVES DE RECHERCHE}

Quelques constats intéressants émergent de notre recherche empirique auprès des Sino-Belges et des Marocains de Belgique que nous voudrions mettre en relation avec la littérature sur le transnationalisme. En premier lieu, la richesse du matériau empirique rassemblé a confirmé l'intérêt d'une approche basé sur les pratiques plutôt que sur les communautés dites transnationales pour deux raisons. D'abord, cette approche nous empêche de verser dans un essentialisme qui nous conduirait à consi- 
dérer certaines communautés comme «naturellement » transnationales tandis que d'autres ne le seraient pas. Cette recherche montre que ni les Marocains ni les Chinois de Belgique, comme du reste aucune autre population, ne peuvent être considérés comme des communautés «naturellement» transnationales. En revanche, des pratiques transnationales diverses peuvent se développer en leur sein de manière circonstancielle. Dès lors, et c'est la seconde raison pour laquelle nous croyons notre perspective féconde, il convient d'expliciter quand, comment et pourquoi une communauté immigrée développe telle ou telle pratique transnationale. Il est possible de proposer quelques pistes de réponses à ces questions. Toutefois, la prudence s'impose si l'on souhaite généraliser au-delà des deux populations étudiées. Il faudrait en réalité poursuivre l'investigation auprès d'autres populations immigrées du pays.

Deux constats surprenants nous conduisent à poursuivre la discussion sur la méthodologie utilisée. Premièrement, notre recherche ne fait pas suffisamment état de la variable "genre » dans les pratiques transnationales économiques, culturelles et sociales. Il faudrait probablement poursuivre la recherche en se focalisant sur la dimension familiale du transnationalisme pour voir émerger le genre comme facteur structurant. Deuxièmement, l'invisibilité de la dimension politique des pratiques transnationales des Chinois correspond à leur invisibilité politique de manière plus générale en Belgique.

En troisième lieu, notre recherche fait bien apparaître que la distinction entre les pratiques économiques, sociales, culturelles et politiques transnationales est incertaine. Elle s'avère utile pour structurer le travail de terrain, mais les pratiques ne correspondent pas parfaitement à cette typologie. Les pratiques économiques peuvent avoir une dimension sociale, culturelle et politique. Les pratiques culturelles ont des implications économiques, etc. Dès lors, si l'usage de ces catégories aux fins de la recherche pouvait se justifier, il convient de ne pas se laisser enfermer dans cette typologie. L'examen simultané de l'ensemble des dimensions des pratiques transnationales de ces groupes devrait idéalement être privilégié.

Cela dit, les pratiques économiques, ou pour être plus précis les préoccupations économiques, des acteurs émergent comme étant les plus importantes et les plus répandues. À nouveau, ce constat doit être lu avec prudence comme émergeant de cette recherche spécifique. Rien ne permet d'affirmer qu'il puisse être généralisé mécaniquement sans recherches ultérieures.

En quatrième lieu, notre recherche confirme l'importance du rôle des États d'origine et d'accueil dans la promotion des pratiques transnationales (Martiniello et Lafleur, 2008). Par exemple, les pratiques économiques transnationales des Chinois de Belgique se développent surtout après les événements de Tienanmen dans les années 1990 lorsque la Chine confirme son choix de l'économie de marché, ce qui ouvre des nouvelles opportunités d'investissement et de profit que les entrepreneurs chinois de l'étranger vont essayer de saisir. C'est à partir de ce moment que les réseaux sociaux au pays d'origine s'avèrent déterminants dans le lancement d'activités transnationales. 
En cinquième lieu, les trois hypothèses suivantes émergent de la recherche de terrain. Premièrement, une bonne intégration économique associée à un sentiment d'appartenance faible au pays de résidence ainsi qu'à une intégration limitée dans son tissu social favorisent les pratiques transnationales, lesquelles peuvent à leur tour se muer ultérieurement en un vecteur d'intégration sociale dans le pays de résidence. Cette hypothèse réfute l'idée selon laquelle les pratiques transnationales seraient un obstacle à l'intégration des immigrés. Deuxièmement, un statut juridique solide dans le pays d'accueil allant jusqu'à l'acquisition de la nationalité favorise les pratiques transnationales. Par la mobilité accrue qu'elle autorise, elle rend possibles certaines pratiques qui ne le seraient pas en l'absence de ce statut de national et donc de citoyen à part entière. Inversement, l'absence de statut juridique réduit la possibilité de mener des pratiques transnationales, à tout le moins, celles qui nécessitent une mobilité physique. À nouveau, ces hypothèses qui émergent d'une recherche exploratoire devront être affinées et testées dans des travaux de terrain avant de pouvoir être généralisées.

\section{Références bibliographiques}

ARROYO ALEJANDRE Jesús et PAPAIL Jean (2005) Les dollars de la migration mexicaine : réinsertion et investissements des migrants internationaux, Paris, L'Haramattan, Collection Travail et mondialisation, $244 \mathrm{p}$.

ATTAR Rachida (1993) « Histoire de l'immigration maghrébine en Belgique », in Anne Morelli, Histoire des étrangers et de l'immigration en Belgique, de la préhistoire à nos jours, Bruxelles, Vie Ouvrière, Collection Histoire, pp. 290-310.

BASCH Linda, GLICK SHILLER Nina et SZANTON BLANC Cristina (1994) Nations Unbound. Transnational Projects, Postcolonial Predicaments and Deterritorialized NationsStates, New York, Gordo and Breach, 330 p.

BAUBOCK Rainer (1994) Transnational citizenship: membership and rights in international migration, Aldershot, Edward Elgar, 348 p.

BELBAH Mustapha et CHATTOU Zoubir (2002) La double nationalité en question. Enjeux et motivations de la double appartenance, Paris, Karthala, Collection Tropiques, 202 p.

BELGUENDOUZ Abdelkrim (2004) «M.R.E» Quelle marocanité ?, Rabat, Imprimerie Beni Snassen, $253 \mathrm{p}$.

BELGUENDOUZ Abdelkrim (2003) Marocains des ailleurs et Marocains de l'intérieur, Rabat, Imprimerie Beni Snassen, $129 \mathrm{p}$.

BENNABOU Mohamed et GARSON Jean-Pierre (1981) «Les Marocains » in Jean-Pierre Garson et Georges Tapinos, L'Argent des immigrés, Paris, PUF, Coll. Travaux et documents, Cahier $n^{\circ}$ 94, pp. 133-171.

BENTON Gregor et PIEKE Frank (Dir) (1997) The Chinese in Europe, Londres, Macmillan Press, $402 \mathrm{p}$.

BLION Raynald et MEKNACHE BOUMAZA Nedjma (Dir) (2005) Europe des migrations/ Europe de développement, Paris, Karthala, Institut Panos, 259 p.

BOUAMAMA Said, CORDEIRO Albano et ROUX Michel (1992) La Citoyenneté dans tous ses états : de l'immigration à la nouvelle citoyenneté, Paris, L'Harmattan-CIEMI, Collection « Migrations et changements », 1992, $361 \mathrm{p}$.

BOUSETTA Hassan et MARTINIELLO Marco (2003) Marocains de Belgique : du travailleur immigré au citoyen transnational, Hommes et Migrations, ${ }^{\circ}$ 1242, pp. 94-106. 
BRIBOSIA Emmanuelle et REA Andrea (Dir.) (2002) Les Nouvelles Migrations : un enjeu européen, Bruxelles, Éditions Complexe, Collection Interventions, 285 p.

CESARI Jocelyne (Dir.) (2002) La Méditerranée des réseaux. Marchands, entrepreneurs et migrants entre l'Europe et le Maghreb, Paris, Maisonneuve et Larose, Collection L'atelier méditerranéen, $300 \mathrm{p}$.

CHAN Sucheng (2005) Chinese American Transnationalism: The Flow of People, Resources, and Ideas between China and America during the Exclusion Era, Philadelphia, Temple University Press, $312 \mathrm{p}$.

CHAREF Mohamed (1999) La circulation migratoire marocaine : un pont entre deux rives, Rabat, Édition Sud Contact, 312 p.

CHATTOU Zoubir (1997) Impact des migrations internes et externes sur les dynamiques locales : le cas de la zone de Berkane, Cahiers du Centre d'études sur les Mouvements Migratoires Maghrébins (CEMMM), Oujda, n 5, pp. 119-138.

CHATTOU Zoubir (1998) Migrations marocaines en Europe : le paradoxe des itinéraires, Paris, L'Harmattan, Collection Histoire et perspectives méditerranéennes, 254 p.

CHEN Zhimin, (2005) Nationalism, Internationalism and Chinese Foreign Policy, Journal of Contemporary China, vol. 14, n42, pp. 35-53.

COLLECTIF (1997) La Belgique et ses immigrés: les politiques manquées, Bruxelles, De Boeck Université, Collection «Politique et histoire », 263 p.

COLONOMOS Ariel (Dir) (1995) Sociologie des réseaux transnationaux, Paris, L'Harmattan, Collection « Dossiers sciences humaines et sociales », 300 p.

FAIST Thomas (2005) The Volume and Dynamics of International Migration and Transnational Social Space, Oxford, Clarendon Press, 380 p.

Fondation Hassan II et O.I.M.(2003) Marocains de l' extérieur, Rabat, Imprimerie Édit, 371 p.

Fondation Hassan II (2005) Marocains de l'extérieur et Développement : Pour une nouvelle dynamique de l'investissement, Rabat, Imprimerie Top Press, 179 p.

GOMEZ Terence (2003) Chinese Enterprise, Transnationalism and Identity Londres, Routledge/Curzon, $240 \mathrm{p}$.

HAMDOUCH Bachir (2000) Les Marocains résidant à l'étranger : une enquête socioéconomique, Rabat, INSEA, $247 \mathrm{p}$.

JACKSON Peter (Dir) (2002) Transnational spaces, Londres, Routledge, 189 p.

KHACHANI Mohamed (2004) Les Marocains d'ailleurs. La Gestion migratoire à l'épreuve du Partenariat Euro-Marocain, Rabat, Imprimerie El Karama, 300 p.

LACROIX Thomas (2005) Les Réseaux marocains du développement. Géographie du transnational et politiques du territorial, Paris, Presses de la Fondation nationale des sciences politiques, Collection Académique, $257 \mathrm{p}$.

LAFLEUR Jean-Michel (2005) Le Transnationalisme politique. Pouvoirs des communautés immigrées dans leurs pays d'accueil et pays d'origine, Louvain-La-Neuve, Academia Bruylant, Collection Cahiers Migrations, $\mathrm{n}^{\circ} 35,76 \mathrm{p}$.

LEUNG Maggi Wai-Han (2004) Chinese Migration in Germany: Making Home in Transnational Space, Francfort, IKO Verlag, $186 \mathrm{p}$.

LI Minghuan (1999) We Need Two Worlds: Chinese Immigrant Associations in a Western Society, Amsterdam, Amsterdam University Press, 360 p.

LIU HUANG Li-Chuan (2008) A Biographical Study of Chinese Restaurant People in Belgium: Strategies for Localisation, Leuven, Katholieke Universiteit Leuven, thèse de doctorat en sciences sociales (non publiée), $391 \mathrm{p}$.

LUTHI Barbara, ZUGIN Bettina et DAVID Thomas (Dir.) (2005) Transnationalisme et migration, Zurich, Chronos, Collection Traverse, $216 \mathrm{p}$.

MA Laurence et CARTIER Carolyn (Dir.) (2003) The Chinese Diaspora: Space, Place, Mobility, and Identity, Lanham MD, Rowman \& Littlefield Publishers, 400 p. 
MARTINIELLO Marco (1992) Leadership et pouvoir dans les communautés d'origine immigrée, Paris, L'Harmattan, Collection Migrations et changements, 317 p.

MARTINIELLO Marco et LAFLEUR Jean-Michel (2008) Towards a Transatlantic. Dialogue in the Study of Immigrant Political Transnationalism, Ethnic and Racial Studies, vol. $30, \mathrm{n}^{\circ} 4$, pp. 645-663.

NYIRI Pal (Dir) (2003) Globalising Chinese Migration: Trends in Europe and Asia Ashgate.

NYIRI Pal et BREIDENBACH Joana (Dir) (2005) Chinese Inside Out: Contemporary Chinese Nationalism and Transnationalism, Budapest, Central European University Press, 354 p.

NYIRI Pal (1999) The New Chinese Migrants in Europe. The Case of the Chinese community in Hungary, Aldershot, Ashgate, $144 \mathrm{p}$.

OCDE (2005) Migrations, transferts de fonds et développement, Paris, OCDE, 385 p.

ONG Aihwa et NONINI Donald (Dir) (1997) Ungrounded Empires: The Cultural Politics of Modern Chinese Transnationalism, Londres, Routledge, 354 p.

OUALI Nouria (Dir.) (2004) Trajectoires et dynamiques migratoires des Marocains de Belgique, Louvain-La-Neuve, Academia Bruylant, Collection Carrefours, 388 p.

PANG Chin Ling (1998) Invisible visibility: intergenerational transfer of identity and social position of Chinese women in Belgium, Asian and Pacific Migration Journal, vol. 7, $\mathrm{n}^{\circ} 4$, pp. 433-452.

PANG Chin Ling (2001) From invisible to unwanted immigrants? The Chinese Communities in Belgium, Migration: European Journal of International Migration and Ethnic Relations, vol. 33, pp. 197-217.

PERALDI Michel (Dir) (2002) La fin des norias ?Réseaux migrants dans les économies marchandes en Méditerranée, Paris, Maisonneuve et Larose, Collection «Série Frontières, villes, lieux de passage », $495 \mathrm{p}$.

PIEKE Frank, NYIRI Pal, THUNO Mette et CECCAGNO Antonella (2004) Transnational Chinese: Fujianese Migrants in Europe, Stanford CA, Stanford University Press, 272 p.

PINA-GUERASSIMOFF Carine (2006) Le renouvellement des perspectives transnationales de la Chine, Paris, Critique Internationale, $\mathrm{n}^{\circ} 32$, pp. 39-52.

PORTES Alejandro (2001), Introduction: the Debates and Significance of Immigrant Transnationalism, Global Networks, vol. 1, n 3, pp. 181-193.

REA Andrea (2001) Jeunes immigrés dans la cité : protestation collective, acteurs locaux et politiques publiques, Bruxelles, Éditions Labor, Collection La Noria, 175 p.

VERMEREN Pierre (2002) La Formation des élites marocaines et tunisiennes. Des nationalistes aux islamistes, 1920-2000, Paris, La Découverte, Collection Recherches, 514 p.

ZHENG Su (2005) Claiming Diaspora. Music, transnationalism and cultural politics in Asian/Chinese America, Oxford, Oxford University Press, 320 p. 


\title{
Les pratiques transnationales des immigrés chinois et marocains de Belgique
}

\author{
Marco MARTINIELLO et Hassan BOUSETTA
}

Cet article s'intéresse aux pratiques économiques, socio-culturelles et politiques transnationales des immigrés marocains et chinois en Belgique. L'objectif central consiste à identifier les caractéristiques et tendances principales des activités menées par ces groupes pardelà les frontières étatiques de leur pays de résidence, de leur pays d'origine et/ou éventuellement d'un pays tiers. Cette contribution s'appuie sur une recherche de terrain et tente d'établir quelques constats comparatifs entre ces deux groupes relativement contrastés. L'article vise ensuite à établir les bases d'une discussion sur la notion de transnationalisme. Les auteurs cherchent à étayer l'idée selon laquelle les conditions structurelles de l'environnement économique, socio-culturel et politique revêtent une importance première par rapport aux variables ethno-culturelles dans l'explication de l'émergence, du maintien et de la reproduction des réseaux transnationaux de ces acteurs issus de l'immigration.

\section{The Transnational Practices of Chinese and Moroccan Immigrants in Belgium}

\section{Marco MARTINIELLO and Hassan BOUSETTA}

This article focuses on the transnational economic, socio-cultural and political practices of Moroccan and Chinese immigrants in Belgium. The main objective is to identify the principal characteristics and trends of the activities carried out by these groups beyond the state borders of, respectively, their country of settlement, of their country of origin and/or possibly a third country. This contribution dwells upon an empirical field research and seeks to establish comparative conclusions about these two contrasted groups. The article then seeks to establish the basis of a discussion on the notion of transnationalism. The authors seeks to support the idea that the structural conditions of the economic, socio-cultural and political environment have a better explanatory power thn ethno-cultural variables in understanding the emergence, maintainance and reproduction of these immigrant transnational actors.

\section{Las prácticas transnacionales de los inmigrantes chinos y marroquíes en Bélgica}

\section{Marco MARTINIELLO y Hassan BOUSETTA}

Este artículo versa sobre las prácticas económicas, socioculturales y políticas transnacionales de los inmigrantes marroquíes y chinos en Bélgica. El objetivo principal consiste en identificar las características y las tendencias principales de las actividades llevadas a cabo por estos grupos más allá de las fronteras estatales de su país de residencia, de su país de origen y/o, en su caso, de un tercer país. Esta contribución se apoya en una investigación empírica e intenta establecer algunas constataciones comparativas entre dos grupos relativamente diferenciados. A continuación, el artículo pretende establecer las bases de un debate sobre la noción de transnacionalismo. Los autores intentan desarrollar la idea según la cual las condiciones estructurales del contexto económico, sociocultural y político revisten una importancia de primer orden con respecto a las variables etnoculturales en la explicación del surgimiento, mantenimiento y reproducción de las redes transnacionales de estos actores procedentes de la inmigración. 